\title{
Présence du caractère autogène chez Culiseta (C.) annulata (Schrank, 1776) et nouvelles mentions géographiques pour Aedes caspius (Pallas, 1771) et Culiseta (C.) subochrea (Edwards, 1921)
}

\author{
R. A. CAPELA et M. E. BRANDAO RIBEIRO
}

Dans un précédent article (R. A. Capela, 1981, sous presse), nous avons signalé la présence de l'autogenèse chez Aedes detritus (Haliday, 1833) du Portugal.

En signalant l'existence du caractère au Portugal, nous apportons une nouvelle contribution à sa répartition géographique et aussi bien à la compréhension de sa valeur physiologique, écologique et systématique.

Nous avons utilisé des femelles vierges et non gorgées issues de larves et de nymphes prélevées dans les gîtes. Les femelles ont été nourries de liquide sucré (miel) et maintenues à $25^{\circ} \mathrm{C}$ et $80 \%$ d'Hr jusqu'à l'émission de la ponte ou à la dissection des ovaires, au $20^{\mathrm{e}}$ jour.

L'état de maturation des follicules ovariens est noté selon l'échelle de Christophers (stades I à V).

Tout ovocyte dépassant le stade III, est considéré comme autogène.

Résultats :

Dans la présente note, l'autogenèse est signalée pour la première fois chez Culiseta annulata (tableau I).

a - Aedes caspius (Pallas, 1771)

O. Theodor (sec. Mattingly, 1953 in : Rioux et coll., 1975) a été le premier à signaler le caractère en Palestine. P. Chinaev (1964) le mit en évidence en Uzbekistan G. A. Babayants et A. B. Karapetyan (1970) en Turkmenistan. J. A. Rioux et coll. (op. cit.) l'ont dépisté dans le sud de la France, au Maroc, en Tunisie et en Grèce.

Muséum et Laboratoire de Zoologie et d'Anthropologie, Faculté des Siences, rue da Escola Politécnica, 1200 Lisbonne, Portugal.

Accepté le $\mathrm{I}_{4}$ novembre $\mathrm{I}_{98} 8_{3}$. 
TABLEAU I.

\begin{tabular}{|c|c|c|c|c|c|c|c|c|c|c|}
\hline \multirow[t]{2}{*}{ Espèce } & \multirow[t]{2}{*}{ Station } & \multirow[t]{2}{*}{ Date } & \multirow[t]{2}{*}{$\begin{array}{c}\text { Nombre de } 9 \\
\text { observées }\end{array}$} & \multicolumn{6}{|c|}{$\begin{array}{c}\text { État de maturation } \\
\text { des ovocytes } \\
\text { (stade de Christophers) }\end{array}$} & \multirow[t]{2}{*}{$\begin{array}{l}\text { Auto- } \\
\text { genèse } \\
\%\end{array}$} \\
\hline & & & & I & II & III & IV & V & pontes & \\
\hline Aedes caspius & $\begin{array}{l}\text { T. Lameiro } \\
\text { L. Paramos } \\
\text { A. Moura } \\
\text { A. Sal }\end{array}$ & $\begin{array}{r}7.3 .1981 \\
16.3 .1981 \\
13.7 .1981 \\
15.7 .1981\end{array}$ & $\begin{array}{r}23 \\
10 \\
4 \\
17\end{array}$ & $\begin{array}{l}1 \\
4 \\
8\end{array}$ & $\begin{array}{r}14 \\
3 \\
3 \\
8\end{array}$ & $\frac{1}{1}$ & $\begin{array}{l}4 \\
- \\
-\end{array}$ & $\begin{array}{r}3 \\
3 \\
- \\
\end{array}$ & $\frac{-}{1}$ & $\begin{array}{r}34,8 \\
30,0 \\
25,0 \\
5,9\end{array}$ \\
\hline Culiseta annulata & $\begin{array}{l}\text { Lisboa } \\
\text { Lisboa } \\
\text { Lisboa } \\
\text { Vagos }\end{array}$ & $\begin{array}{l}15.12 .1980 \\
15.1 .1981 \\
18.2 .1981 \\
17.3 .1981\end{array}$ & $\begin{array}{l}43 \\
81 \\
59 \\
36\end{array}$ & $\begin{array}{r}6 \\
3 \\
1 \\
12\end{array}$ & $\begin{array}{l}20 \\
29 \\
45 \\
21\end{array}$ & $\begin{array}{r}4 \\
29 \\
6 \\
1\end{array}$ & $\begin{array}{r}3 \\
10 \\
5 \\
2\end{array}$ & $\begin{array}{r}10 \\
10 \\
2 \\
-\end{array}$ & E & $\begin{array}{r}39,5 \\
60,5 \\
22,0 \\
8,3\end{array}$ \\
\hline Culiseta subochrea & $\begin{array}{l}\text { A. Moura } \\
\text { L. Albufeira }\end{array}$ & $\begin{array}{l}22.2 .1980 \\
28.1 .1981\end{array}$ & $\begin{array}{l}35 \\
18\end{array}$ & $\begin{array}{r}18 \\
6\end{array}$ & $\begin{array}{r}5 \\
10\end{array}$ & 5 & 1 & $\begin{array}{l}6 \\
2\end{array}$ & 二 & $\begin{array}{l}34,3 \\
11,1\end{array}$ \\
\hline
\end{tabular}

Nous l'avons observé en 1981 dans le Centre et au Nord du Portugal. Le caractère autogène est présent chez Aedes (O.) caspius et ce, à des taux variables suivant la période d'observation.

b - Culiseta annulata (Schrank, 1776)

L'autogénèse est signalée pour la première fois (fig. 1 et 2) chez cette espèce, présente dans la majeure partie de la région Paléarctique. Les femelles étaient issues de larves et de nymphes récoltées dans le Jardin des Plantes de Lisbonne et à Rio Maior, dans des gîtes (roubines) situés à l'ombre d'arbres de grande taille. L'eau était douce $(0,2 \mathrm{~g} / \mathrm{l} \mathrm{du} \mathrm{NaCl})$, le $\mathrm{pH}$ de 6,0 . La fréquence du caractère étudié en décembre 1980, janvier, février, mars 1981 a été relativement élevée, sauf en mars.

c-Culiseta subochrea (Edwards, 1921)

Chez cette espèce, la présence du caractère a été signalée pour la première fois par J. F. Marshall (1936). A. B. Maslov (1962) mentionne le phénomène de Russie septentrionale. G. A. Babayants et A. B. Karapetyan (op. cit.) le retrouvent au Turkmenistan. J. A. Rioux et coll. (1974) l'ont dépisté en Tunisie et dans le Sud de la France (1975).

Nous l'avons observé à partir de femelles issues de larves et de nymphes des gîtes (rizières) des environs d'Aguas de Moura.

La fréquence du caractère étudié en février $1980(34,3 \%)$ et janvier $1981(11,1 \%)$ a été moyenne par rapport aux valeurs trouvées en février par J. A. Rioux et coll. (op. cit.) en Tunisie (64\%) et en Camargue (93\%), 1975.

Nous voulons remercier M. le Professeur J.-A. Rioux qui a bien voulu critiquer ce travail. 


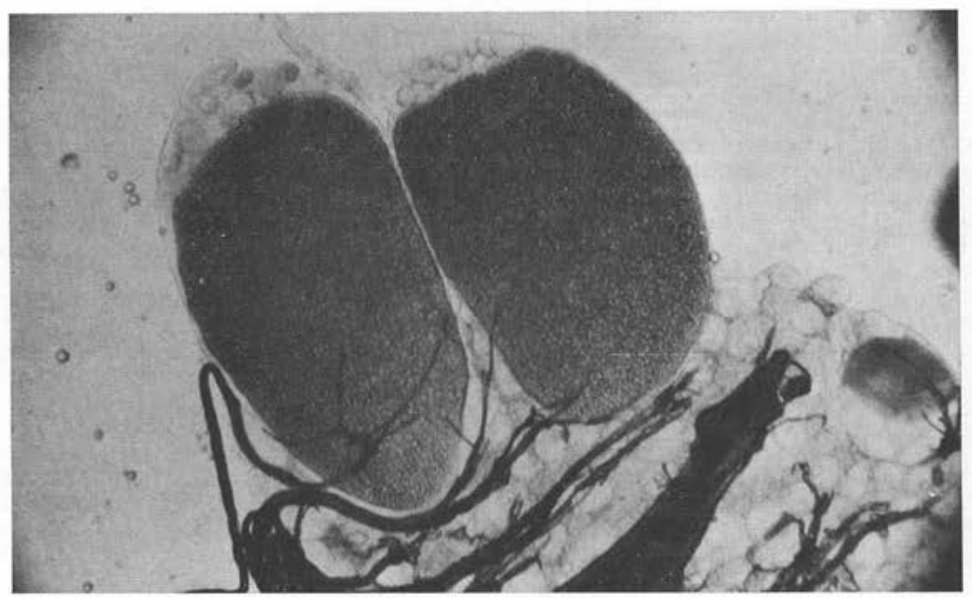

FIG. I. - Ovaire de Culiseta annulata. Dissection au $20^{\mathrm{e}}$ jour.

Stade IV de Christophers. Au voisinage, les autres ovocytes restent bloqués aux stades I-II.

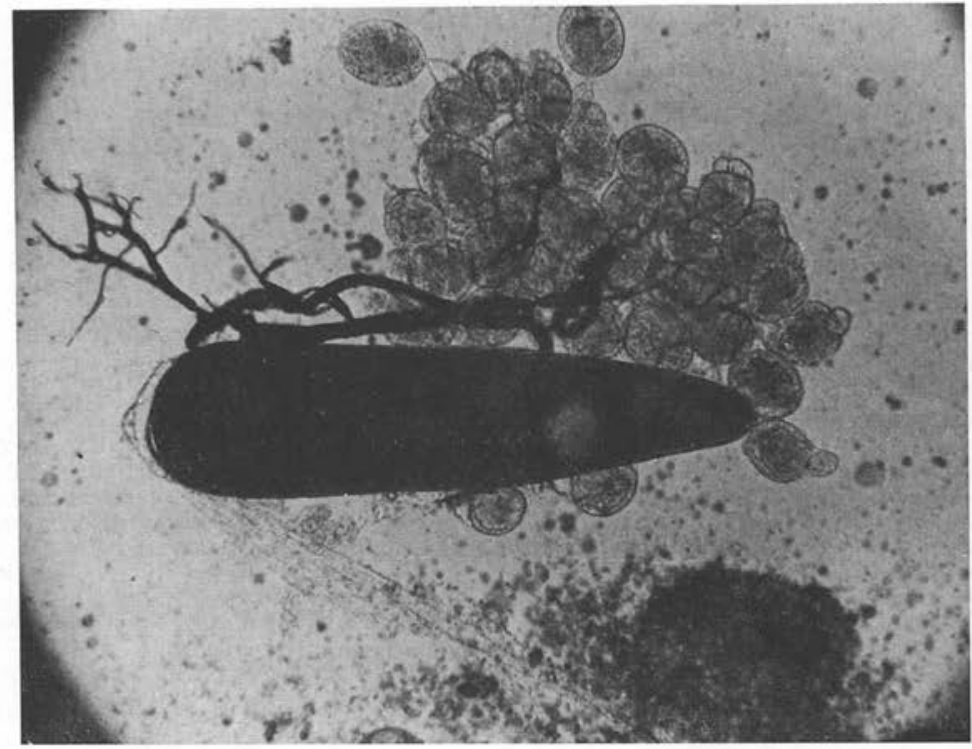

FIG. 2. - Ovaire de Culiseta annulata. Dissection au $20^{\mathrm{e}}$ jour.

Un œuf mûr, prêt à être pondu (stade V). Tous les autres ovocytes restent bloqués au stade II. 


\section{BIBLIOGRAPHIE}

Babayants G. A., Karapetyan A. B. : Développement autogène des ovaires chez certaines espèces de moustiques de Turkménie. Med. Parazit., 1970, 39, 24-29.

CAPELA R. A. : Présence de caractères autogènes et sténogame dans les populations d'Aedes $(O$. detritus (Haliday, 1833) de la Côte Sud (Algarve) du Portugal. I981 (sous presse).

ChINAEV P. P. : On the autogenous development of exophilous mosquitoes in Uzbequistan. Zoll. $Z h ., 1964,43,939-940$.

Marshall Y. F., STALEY Y. : Exhibition of autogenous and stenoganous charateristics by Theobaldia subochrea Edwards (Diptera, Culicidae), Nature. 1936, 137, 580.

Maslov A. B. : Les moustiques hématophages. Culisetina (Diptera, Culicidae). Acad. Sc. Léningrad. U.R.S.S., 1967,181 p.

Rioux J. A., Kchouk M., Croset H., Ben Osman F., Belmonte A. : Présence du caractère autogène dans les populations tunisiennes de Culiseta (C.) subochrea (Edw., I92I) (Diptera, Culicidae). Ann. Parasitol. Hum. Comp., 1974, 49, 371-373.

Rioux J. A., Cousserans J., Croset H., Ben Osman F., Gabinaud A., Sinegre G., Belmonte A.: Présence du caractère autogène chez Aedes pullatus (Coquillett, 1904) et nouvelles localisations géographiques pour Aedes caspius (Pallas, I77I), Aedes mariae (Sergent et Sergent, 1903). Aedes detritus (Haliday, 1833) et Culiseta subochrea (Edwards, 1921). Ann. Parasit. Hum. Comp., 1975, so, 131-142.

Des Microfiches et des MicrofiLms de cette publication PEUVEnt Être obtenuS Auprès de :

This publication is available in Microform from :

MASSON - SPPIF (réf. MIMC)

120, boulevard Saint-Germain, 75280 PARIS CEDEX o6

(C) Masson, Paris, 1984 .

Le Directeur de la Publication: Dr J. Talamon

Tous droits de traduction, d'adaptation et de reproduction par tous procédés véservés pour tous pays

La loi du II mars I957 n'autorisant, aux termes des alinéas 2 et 3 de l'article 41 , d'une part, que les $\alpha$ copies ou reproductions strictement réservées à l'usage privé du copiste et non destinées à une utilisation collective " et, d'autre part, que les analyses et les courtes citations dans un but d'exemple et d'illustration, " toute représentation ou reproduction intégrale ou partielle, faite sans le consentement de l'auteur ou de ses ayants droit ou ayants cause, est illicite " (alinéa I ${ }^{\text {er }}$ de l'article 40).

Cette représentation ou reproduction, par quelque procédé que ce soit, constituerait donc une contrefaçon sanctionnée par les articles 425 et suivants du Code pénal.

Masson, éditeur, Paris. - Dépôt légal : 1984 . -

IMPRIMERIE DARANTIERE DIJON-QUETIGNY (FRANCE). -
No d'ordre : 5505 . - Ostobre 1984

Commission paritaire no 54169

Printed in France. 